\title{
CAE analysis of display module under impact failure
}

\author{
Sa-yoon Kim ${ }^{1}$, Myeong-woo Lee ${ }^{1}$, Hyun-woo Jung ${ }^{1}$, Yun-jae Kim ${ }^{*}$, and Hye-young Kim ${ }^{2}$ \\ ${ }^{1}$ Mechanical Engineering, Korea University, 145, Anam-ro, Seongbuk-gu, Seoul, Korea \\ ${ }^{2}$ Quality \& Reliability Team, Samsung Display, 1, Samsung-ro, Giheung-go, Yongin-si, Gyeonggi-do, Korea \\ ${ }^{*}$ Corresponding author’s email: kimy0308@korea.ac.kr
}

\section{Introduction}

When a mobile phone gets impact by dropping, failure may occur due to disconnection of the display module wiring. To figure out the failure modes accurately, FE analysis can be one of essential method. After developing CAE analysis method, failure modes of mobile phone by impact will be defined and valuation criteria will be proposed as well.

In order to develop analysis method, it is necessary to understand detail shape on inside of mobile phone by teardown. Then it needs to be classified into significant and insignificant parts for simplifying the model. Shell element will be used on model for efficient analysis after verifying on simple model. In addition, drop test will be performed with experimental apparatus that has been designed. With comparison of acceleration and displacement after impact, consistency between CAE model and experiment data, will be verified.

\section{Pre-analysis for shell element}

Since shell element is necessary for efficient analysis, verification on simple model was performed. For dynamic analysis, figure 1 shows deflection graph on cantilever model that has impact with $10 \mathrm{~m} / \mathrm{s}$ on middle point. The result from 0.15 of mesh size and C3D20 element type was considered as reference value, and material was STS 410. The result implies that gap between reference and solid analysis value, becomes smaller when size of mesh becomes smaller while shell element does not. Difference of error percentage is around $2 \%$ which means there is no significant difference for using shell element unless size of mesh is highly small. Through these verification, shell element has been proven to be used under certain size of mesh.

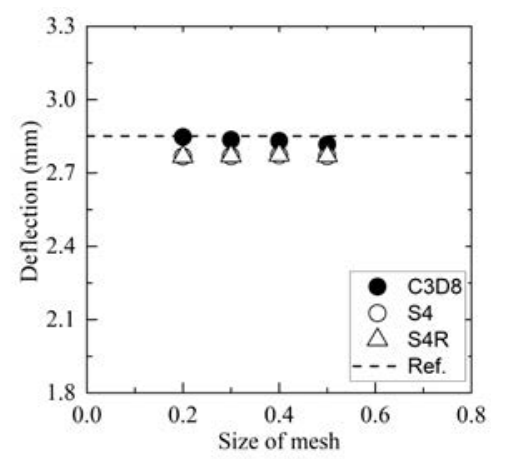

Fig. 1 Dynamic analysis of solid/shell element

\section{Modeling}

With teardown, drawing, and 3D scanning, shape information was acquired on I-phone for modeling. Figure 2 shows modeling of I-phone and impact force after FE analysis. Any parts which of weight is under $5 \mathrm{~g}$, was excluded and reflected on SUS plate for density change. Element type was shell element $\mathrm{S} 4$, and sizes of mesh were $0.1 \mathrm{~mm}$ on impact region and upto $5 \mathrm{~mm}$ on insignificant part respectively. FE analysis was performed with double precision on Abaqus explicit, and $5.5 \mathrm{~m} / \mathrm{s}$ of initial velocity and gravity were applied to realize a free fall from $1.5 \mathrm{~m}$. The impact surface of floor which of material is stainless steel, has similar mesh size to impact surface of specimen. Impact force, deflection, and acceleration are parameters to understand behavior of I-phone.
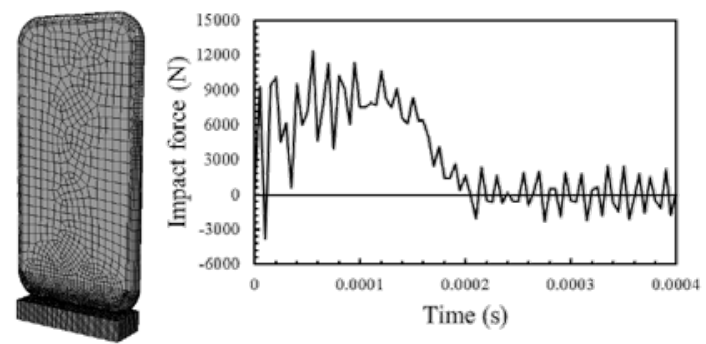

Fig. 2 I-phone modeling and impact force

\section{Experiment}

An experimental apparatus was designed to confirm consistency of FE analysis. The mobile phone hung by 4 strings, is able to be adjusted with angle and shows independent behavior without effect of guide rail just after impact. An electromagnet was used to control the start of fall, and impact surface which located bottom of apparatus was designed to be replaced for various impact materials. A load cell is located right under the impact material and an accelerometer is attached on mobile phone. Both of data of impulse and acceleration change can be extracted at a time.

\section{Conclusion}

Through comparison between FE analysis and experimental data, reliability from them is acquired. For further study from FE analysis, it can be realized to understand behavior after impact then propose better alternatives for design. 